\title{
Application of simulated annealing to inverse design of transonic turbomachinery cascades
}

\author{
W T Tiow*, K F C Yiu and M Zangeneh \\ Department of Mechanical Engineering, University College London, UK
}

\begin{abstract}
In turbomachinery blade design, inverse methods and optimization techniques are often applied independently to produce high performance blade shapes. The idea of using an optimization algorithm to seek the optimal target distribution for an inverse design methodology has been explored. However, these efforts have been made mainly in the design of single aerofoils. In this paper, a new inverse design method is coupled with a simulated annealing algorithm to search for the optimum turbomachinery cascade shape. In order to speed up the algorithm, a database of generated designs is set up and the nearest match is selected to initialize subsequent calculation. The proposed computational procedure equips engineers with an automatic design tool with which the inverse method may be applied in isolation or combined with the optimization algorithm to produce the optimum.

The inverse methodology is based on a cell vertex, finite volume time-marching flow solver that gives the viscous cascade flow solution in both the subsonic and the transonic flow regimes. The cascade shape is computed subject to an imposed distribution of the mass-averaged tangential velocity and a specified tangential thickness profile. The solver code is validated using experimental data and the accuracy of the inverse method is verified by regenerating a known cascade geometry starting from a different one using its mass-averaged tangential velocity distribution. In combining the inverse methodology with the optimization algorithm, the mass-averaged tangential velocity distribution is parametrized using a cubic $B$-spline curve and the proposed simulated annealing algorithm is applied to predict the optimal distribution by minimizing loss. The overall procedure is demonstrated to produce optimum shapes of a transonic axial turbine and an axial compressor rotor.
\end{abstract}

Keywords: turbomachinery cascade, inverse design, optimization, simulated annealing

\section{NOTATION}

$C_{\mathrm{f}} \quad$ skin friction coefficient

$C_{\mathrm{p}} \quad$ pressure coefficient

E total energy

$f \quad$ camber line

$F \quad$ frictional force

H flow properties

$l \quad$ surface arc length

$m \quad$ meridional distance

$N \quad$ blade number

$P \quad$ static pressure

$r \quad$ radius

$r V_{\theta} \quad$ swirl velocity (design parameter in three dimensions)

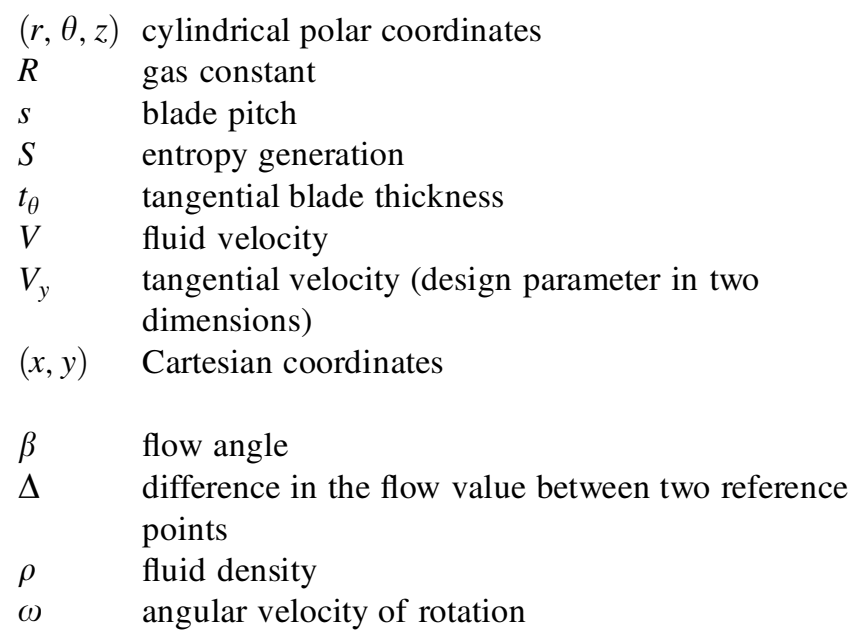

\section{Superscripts}

$0 \quad$ initial value

1 first iteration
The MS was received on 10 November 2000 and was accepted after revision for publication on 16 July 2001.

*Corresponding author: Department of Mechanical Engineering, University College London, Torrington Place, London WC1E 7JE, UK. 
$n \quad n$th iteration

$+\quad$ upper surface

- lower surface

* required/target value

\section{Subscripts}

bl blade average value

m meridional component

$x \quad$ horizontal component

$y \quad$ vertical component

$\theta \quad$ tangential component

0 stagnation value

1 cascade inlet

2 cascade outlet

\section{Overbars}

$\begin{array}{ll}\sim & \text { mass average } \\ -\quad & \text { pitch average }\end{array}$

\section{INTRODUCTION}

Computational techniques are now routinely applied in the field of turbomachinery. In the development of turbomachinery blades, a variety of specialized computer codes are required to assess and modify a design before the prototype is made. Consequently, the availability of good analysis and design codes is vital for manufacturers to stay at the forefront of a very competitive field of engineering. Inverse methodologies and practical automatic optimization procedures provide a systematic means of design, reducing the considerable time and cost often incurred in the conventional way of iterating between analysing the design and modifying the blade shape manually.

Many two-dimensional inverse design methods are available, such as those in references [1] to [3], and are commonly used in the preliminary stage of the design process for axial turbomachines. In recent years, threedimensional inverse design methods have emerged and been applied successfully for a wide range of designs, involving both turbomachinery blades and wings [4-6].

In this paper, a new inverse design methodology coupled with a simulated annealing (SA) optimization algorithm is presented for the design of optimum axial blade cascades in transonic viscous flow. The inverse method is based on the flow governed by the Euler equations of motion and improved with viscous effects modelled using a body force model as given by Denton [7]. In the proposed inverse design methodology, the design specification is the massaveraged swirl velocity $\left(r \tilde{V}_{\theta}\right.$, i.e. radius multiplied by tangential velocity) and the thickness distribution. The method is therefore different from previous Euler-based inverse methods proposed by Leonard and Van Den Braembussche [3], Demeulenaere and Van Den Braembussche [5] and Giles and Drela [8], who specified the static pressure distribution on the upper and lower surfaces of the blades, or Dang and Isgro [9], who specified the circulation distribution for the design of infinitely thin blades. Recently, methodologies based on the specification of static pressure loading $(\Delta P)$ which apply the transpiration model for the blade updates were reported $[\mathbf{1 0}, \mathbf{1 1}]$. By specifying the $\Delta P$ distribution together with the tangential thickness, it is possible to control the blade pressure loading directly, which is very important in controlling shock losses in transonic/supersonic flow and cavitation in incompressible flow, while maintaining structurally sound blades. However, the difficulty in using methods based on $\Delta P$ is that the specific work is not directly controlled. The method in reference [11] for example, is implemented together with a secant loop to fulfil a specific work requirement in an iterative manner. In this paper, an inverse methodology is presented capable of providing designs directly for a specific work using the mass-averaged swirl velocity $r \tilde{V}_{\theta}$ distribution. The $r \tilde{V}_{\theta}$ distribution is used as the design specification since its use can be easily extended to three dimensions and its meridional derivative $\partial r V_{\theta} / \partial m$ is directly related to the difference in static pressure across the blade (see equation (4)). This type of design specification has already been applied in many turbomachinery designs, such as in the suppression of secondary flows in centrifugal impellers [12], improving pump suction performance [13] and designing high performance propellers $[\mathbf{1 4}, \mathbf{1 5}]$.

The current design procedure consists mainly of a flow analysis code and a blade update module coupled together to give the required cascade shape and the corresponding flow field. No modifications are needed in the flow solver when used in the design process. There is therefore no difference in the solver code when used in either the 'design' or the 'analysis' mode. This is a major difference from existing inverse design techniques based on the transpiration model where the flow solver code has to be modified to model the blade walls as permeable. The three-dimensional application of this new methodology has recently been reported by Tiow and Zangeneh [16] in the design of a transonic fan blade.

From the ongoing work of the authors, it has emerged that, for the full potential of the inverse design methodology to be exploited, it is important for the optimum design specification to be sourced in a systematic and efficient manner. This vital task currently relies heavily on the experience of the engineers who are using the codes. The prospect of seeking the optimal design distribution via optimization techniques has been identified by Van Egmond and colleagues $[\mathbf{1 7}, \mathbf{1 8}]$ for the inverse design of aerofoils. This line of research has also been followed by Yiu and Zangeneh [19] for impeller blade design, where the swirl velocity distribution is parametrized by a cubic $B$-spline curve via several control points and an automatic optimization algorithm is invoked to minimize the loss with respect to the control points. In the current development, a similar approach is adopted.

Traditional optimization methods such as the gradient technique can identify local minima quickly but cannot 
reach the global minimum easily. Since aerodynamic loss is a highly non-linear function of the blade shape, many local minima could exist. Therefore, a global minimization technique should be applied. Exploratory techniques avoid focusing only on a local region and their stochastic nature generally evaluates designs throughout the parameter space in search of the global optimum. Genetic algorithms (GAs) form one class of such techniques. These techniques are essentially search algorithms based on the mechanics of natural selection in the evolution of populations, and as such they are highly demanding in terms of computational requirement and are often too expensive when applied to shape design. Another exploratory technique is Simulated Annealing (SA). It can be applied for finding the global or near-global optimum solutions of large and difficult problems, like that of the travelling salesman [20], and is a more economical stochastic method in terms of computational time compared with GA methods [21]. On the basis of these considerations, SA is chosen in the proposed design procedure. The term 'annealing' refers to the process in which a solid material is first heated and then allowed to cool by slowly reducing the temperature. If the solid is cooled too quickly, it will not reach the global minimum state of its potential energy function. In nature, the energy states of a system follow the so-called Boltzmann probability distribution, which expresses the idea that a system in thermal equilibrium at temperature $T$ has its energy probabilistically distributed among all different energy states. Metropolis et al. [22] were the first to incorporate this principle into numerical calculations by employing the Monte Carlo method to simulate the evolution to thermal equilibrium of a solid at a fixed temperature. This idea has been extended further to form the basis of the SA algorithm first proposed by Kirkpatrick et al. [20] and independently by Cerny [23].

In the current design procedure, the cost function is defined by the maximum mass-averaged loss along the streamwise direction. The swirl velocity distribution is parametrized by a cubic $B$-spline curve and a biased random walk in the space of feasible spline control points is implemented to search for the minimum cost function. During the search, some or all of the spline control points are randomly perturbed and their acceptance depends on a Boltzmann probabilistic distribution. The perturbation process is repeated until a predefined number of iterations are reached. Because of the stochastic property, the longer the algorithm is executed, the closer the global minimum is approached. However, for each iteration, the corresponding flow field needs to be evaluated. To speed up this process, generated designs and their flow fields are stored in a database and the nearest result within the database is used to initialize the flow calculation of the next iteration. Using this approach, the required running time is shortened considerably, with increasing gain in speed as a larger database is accumulated during the process. The proposed design method is demonstrated in the design of a compressor and a turbine cascade. These test cases are generic but they exhibit flow field features typically encountered in practical transonic conditions. Using these examples, complicated transonic features like strong shock formation and boundary layer build-up (or diffusion) are shown to be effectively controlled/reduced using the presented procedure.

\section{EULER FLOW SOLVER}

The flow solver computes the solution of the unsteady Euler flow equations through a rotating turbomachine blade row. The equations satisfy continuity, momentum and conservation of energy with assumptions of the flow medium as an ideal gas. In two dimensions, the equations cast in Cartesian coordinates $(x, y)$ are as follows:

$$
\begin{aligned}
& \frac{\partial}{\partial t} \iint \mathbf{H} \mathrm{d} x \mathrm{~d} y+\oint(\mathbf{E} \mathrm{d} y-\mathbf{F} \mathrm{d} x)=\mathbf{S}_{\mathrm{vis}} \\
& \mathbf{H}=\left[\begin{array}{c}
\rho \\
\rho V_{x} \\
\rho V_{y} \\
\rho E
\end{array}\right], \quad \mathbf{E}=\left[\begin{array}{c}
\rho V_{x} \\
\rho V_{x}^{2}+P \\
\rho V_{x} V_{y} \\
\rho V_{x} h_{0}
\end{array}\right], \\
& \mathbf{F}=\left[\begin{array}{c}
\rho V_{y}+r \omega \rho \\
\rho V_{x} V_{y}+r \omega \rho V_{x} \\
\rho V_{y}^{2}+P+r \omega \rho V_{y} \\
\rho V_{y} h_{0}+r \omega \rho E
\end{array}\right], \quad \mathbf{S}_{\mathrm{vis}}=\left[\begin{array}{c}
0 \\
F_{x} \\
F_{y} \\
0
\end{array}\right]
\end{aligned}
$$

The solver works with absolute flow quantities and the conservation equations are applied to a fixed grid, which instantaneously coincides with the rotating grid on which the solution is required. Equations in the rotating framework are formulated to model the rotating cascades and the implementation follows closely that suggested by Denton and Singh [24]. Blade rotation is simulated by the terms $r \omega \mathbf{H}$ given in the matrix $\mathbf{F}$ where $r \omega$ is set as a constant to represent the blade speed. The source term, $\mathbf{S}_{\mathrm{vis}}$, represents frictional force components due to viscosity in the flow. Descriptions of the viscous model are given in the next section.

The unsteady flow equations are discretized and solved for steady solution. The approach utilizes a cell vertex, finite volume [25], central difference scheme with added dissipation and a Runge-Kutta integrator proposed by Jameson et al. [26].

The developed solver has blended non-linear second- and fourth-difference artificial dissipation added explicitly to suppress spurious oscillations and for accurate capturing of shock formations. It is noted that the use of local time stepping is implemented in the present computer code for convergence acceleration.

In the cascade flow calculation, four different types of boundaries are required: inlet, outlet, solid walls and periodicity. At the inlet, the total pressure and temperature and 
the absolute flow direction are specified. The static pressure is extrapolated from the interior flow field domain, which is used in conjunction with the isentropic relation to calculate the density and velocity.

At the downstream boundary, the exit static pressure is fixed. The remaining flow variables are then extrapolated from those at the interior nodes. At the solid surfaces of the cascade, normal convective fluxes along the solid walls are forced to zero, and the surface flow satisfies the tangency condition and slips along the blade surfaces. A periodic or a cyclic condition is imposed to model the symmetry of flow between each sector of the cascade. This condition is applied upstream of the leading edge and downstream of the trailing edge where the values at two corresponding points are set to be equal.

\subsection{Modelling of viscous effects}

Viscous effects are modelled as proposed by Denton [7]. The effects are implemented by calculating the surface shear stresses and then distributing them to give the components of viscous forces acting on each control volume. The viscous force components are included in the flow formulation as source terms in the right-hand side of each of the momentum equations as shown in equations (1). The surface shear stresses are estimated via a skin friction factor, $C_{\mathrm{f}}$, and distributed via a power law variation. The reader is referred to the original article [7] for more details.

\subsection{Validation}

The solver code has been validated extensively. One validation in two dimensions is performed using the mid-span section of a UTRC turbine rotor blade row [27]. The cascade is computationally modelled with simple sheared $\mathrm{H}$ grid (see
Fig. 1) with 91 nodes in the streamwise and 21 in the pitchwise direction. Pitchwise grid points are non-uniformly spaced with finer mesh nearer the blade surfaces. The computed distributions of the coefficient of pressure, $C_{\mathrm{p}}$, along the pressure and suction sides of the profile are compared against the given experimental data.

Figure 2 shows the comparison between the experimental values and the numerical solution. With the exception of minor discrepancies near the leading and trailing edges, the correlation is satisfactory.

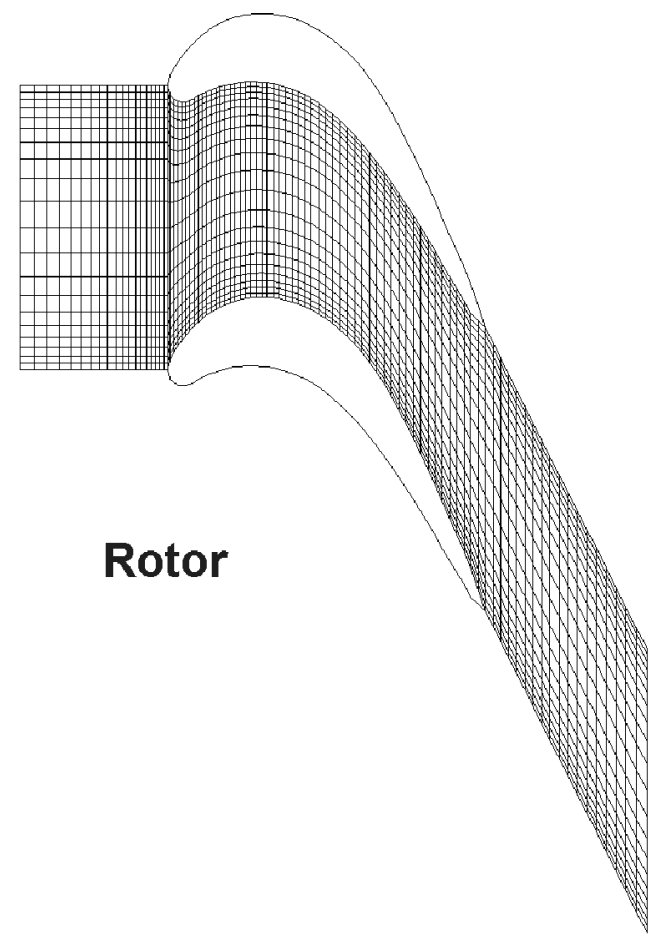

Fig. 1 Simple H mesh for UTRC rotor cascade

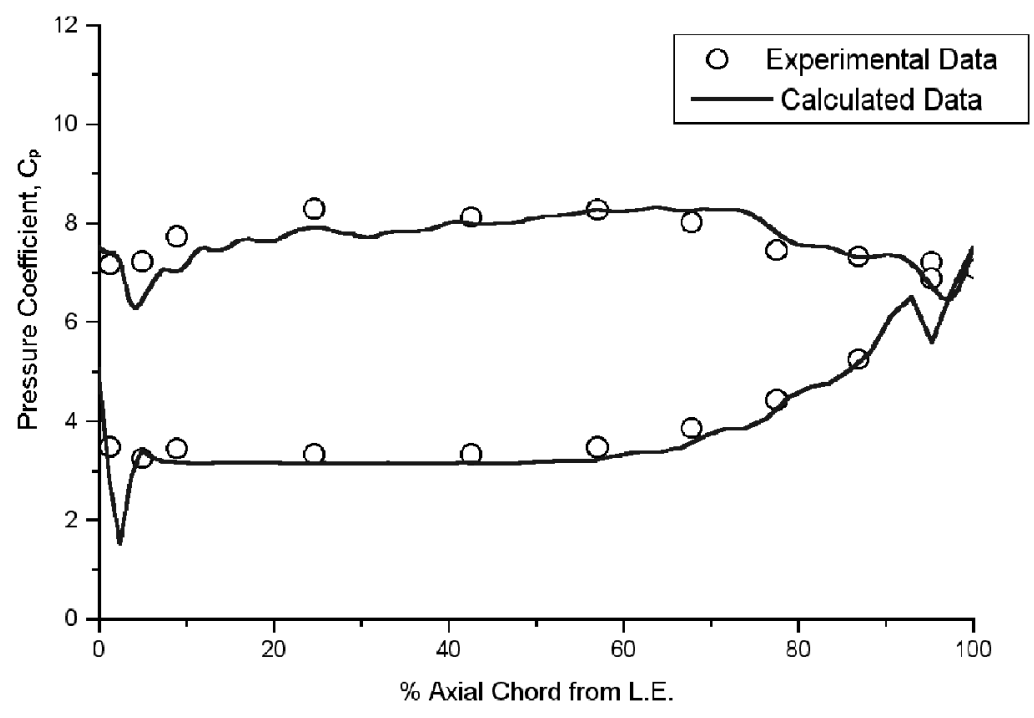

Fig. 2 Validation: UTRC rotor. Comparison of numerical and experimental pressure coefficients, $C_{\mathrm{p}}$ 


\section{THE INVERSE DESIGN METHODOLOGY}

In the inverse design procedure, the solver code is coupled with the blade design algorithm. The solution of the new blade geometry is determined directly from the difference between the target and currently obtained $r \tilde{V}_{\theta}$ distribution provided by the flow solver.

The inverse design cycle consists of two main steps (see Fig. 3):

1. The first step determines the new geometry and generates the corresponding computational mesh. The blade update algorithm is based on the flow tangency condition, which is satisfied along the surfaces of the blade profile. The discrepancies between the specified mass-averaged tangential velocity and the actual distribution determine the geometrical modification needed for the current geometry.
2. The second step carries out the analysis of the flow field in the new computational domain. Every blade update is accompanied by a corresponding regeneration of the computational mesh. To alleviate the computational time and effort in the generation of mesh following each blade modification, a simple $\mathrm{H}$ grid is used to represent the computational domain.

\subsection{Design specification}

The prescribed quantity, circumferential mass-averaged tangential velocity $r \tilde{V}_{\theta}$, is defined as

$$
r \tilde{V}_{\theta}=\frac{\int_{\mathrm{LS}}^{\mathrm{US}} r V_{\theta}\left(\rho V_{\mathrm{m}}\right) \mathrm{d} \theta}{\int_{\mathrm{LS}}^{\mathrm{US}} \rho V_{\mathrm{m}} \mathrm{d} \theta}
$$

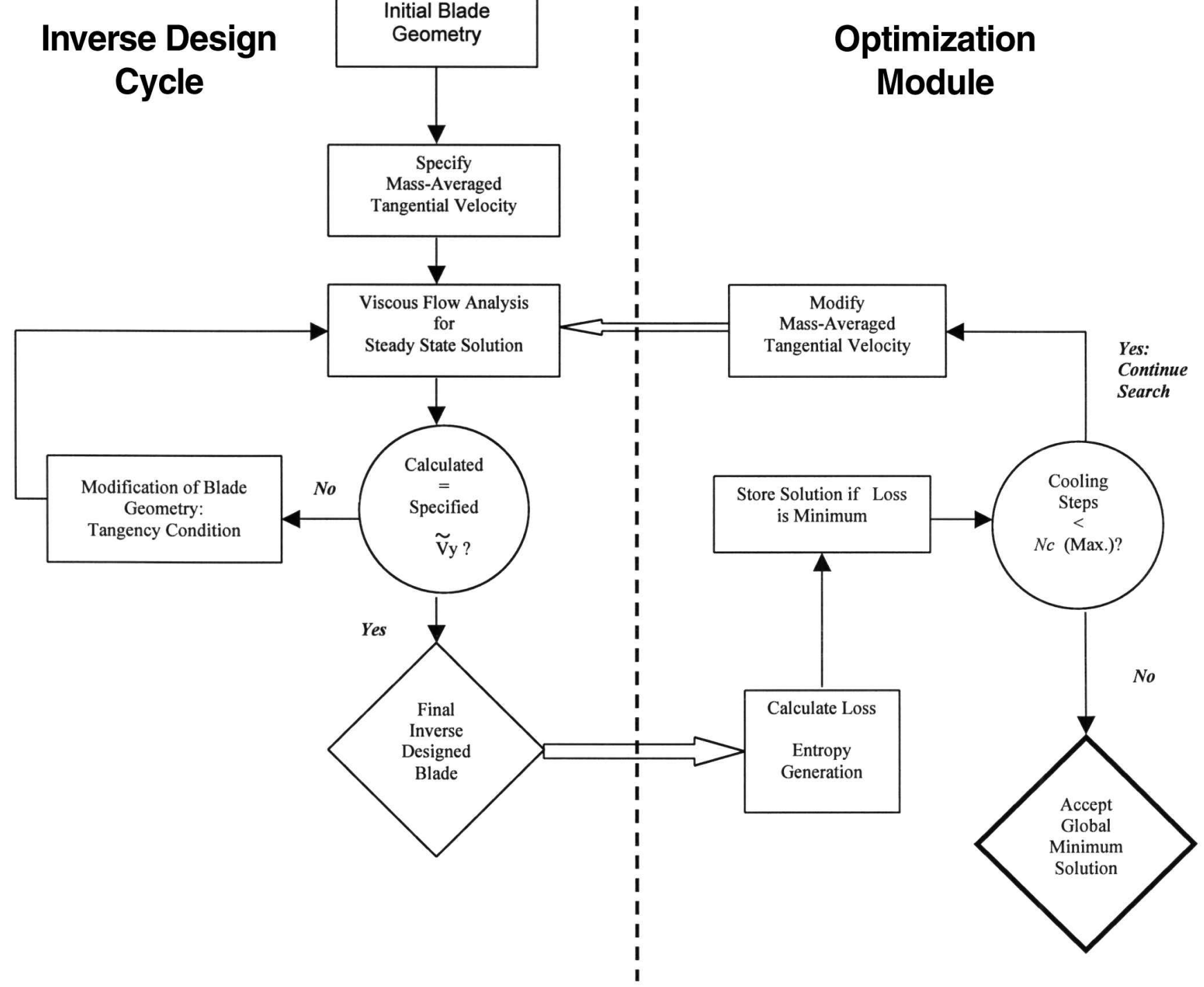

Fig. 3 Proposed inverse optimization procedure 
where the limits US and LS denote the upper and lower side of the cascade and subscripts $\theta$ and $\mathrm{m}$ represent the circumferential and meridional direction respectively.

The specification of the $r \tilde{V}_{\theta}$ distribution is not arbitrary. The Euler equation for turbomachinery states that

$$
\dot{W}=\omega\left[\left(r V_{\theta}\right)_{2}-\left(r V_{\theta}\right)_{1}\right]
$$

where $\left(r V_{\theta}\right)_{1}$ and $\left(r V_{\theta}\right)_{2}$ are the swirl velocity quantities at the inlet and outlet of the cascade respectively. It is therefore clear that by imposing the fluid swirl velocity, the design of the blade geometry may be performed directly on the basis of a required specific work, $\dot{W}$, expected of the machine.

The rate of change of the swirl velocity along the blade is also related to the difference in static pressure loading (or $\Delta P)$. In fact, for a thin blade in incompressible, irrotational flow, reference [28] shows that the pressure jump across the blade, $\Delta P$, is related to the tangentially averaged swirl velocity $r \bar{V}_{\theta}$ :

$$
\Delta P=P^{+}-P^{-}=\frac{2 \pi}{N} \rho V_{\mathrm{m}_{\mathrm{bl}}} \frac{\partial r \bar{V}_{\theta}}{\partial m}
$$

where $V_{\mathrm{m}_{\mathrm{b}}}$ is the meridional velocity at the blade surface, $\partial r \bar{V}_{\theta} / \partial m$ is the derivative of the swirl velocity quantity in the meridional direction and the superscripts,+- represent the upper and lower blade surfaces respectively. Thus, the imposition of the swirl velocity values at each point along the blade not only allows the design to be based directly on the specific work required by the blade but also allows the design to follow a specific blade pressure loading trend.

In two dimensions, the mass-averaged tangential velocity, $r \tilde{V}_{\theta}$, is equivalent to the mass-averaged $y$-directed velocity (or tangential velocity), $\tilde{V}_{y}$. Following equation (2), the definition of the design parameter in two dimensions is therefore

$$
\tilde{V}_{y}=\frac{\int_{\mathrm{LS}}^{\mathrm{US}} V_{y}\left(\rho V_{x}\right) \mathrm{d} y}{\int_{\mathrm{LS}}^{\mathrm{US}} \rho V_{x} \mathrm{~d} y}
$$

The next section shows the theoretical development of the design concept in two dimensions using this parameter directly.

\subsection{Camber line generator}

The discrepancy between the specified and obtained massaveraged tangential velocity determines the amount of modification needed for the current geometry. It remains to be shown how the prescribed values, $\tilde{V}_{y}^{*}$, are used to modify the initial blade geometry defined with camber $f^{0}$.

The blade surfaces are represented by

$$
\alpha^{ \pm}=y-f^{ \pm}(x)=n s, \quad \text { where } n=0,1,2, \ldots, \infty
$$

where $s$ is the blade pitch and $f^{ \pm}(x)$ represents the upper and lower surfaces of the two-dimensional blade cascade (see Fig. 4). Along the solid walls of the cascade, the flow satisfies the tangency condition (wall boundary condition, Section 2). Mathematically, the condition can be expressed as

$$
\boldsymbol{V}^{ \pm} \cdot \nabla \alpha^{ \pm}=0
$$

where $\boldsymbol{V}^{ \pm}$represents the velocity vectors on the upper and lower surfaces of the cascade.

If it is noted that

$$
f^{ \pm}=f \pm \frac{t_{\theta}}{2}
$$

where $f$ is the camber line, equation (7) can be expressed as

$$
V_{x}^{ \pm} \frac{\mathrm{d}\left(f \pm t_{\theta} / 2\right)}{\mathrm{d} x}=V_{y}^{ \pm}
$$

Then, on applying the equations to the initial camber, $f^{0}$, and adding, the expression becomes

$$
\left(V_{x}^{+0}+V_{x}^{-0}\right) \frac{\mathrm{d} f^{0}}{\mathrm{~d} x}+\frac{\mathrm{d} t_{\theta}}{\mathrm{d} x}\left(\frac{V_{x}^{+0}-V_{x}^{-0}}{2}\right)=V_{y}^{+0}+V_{y}^{-0}
$$

where the superscript 0 corresponds to the current flow field computed for the cascade shape having the camber $f^{0}$.

\section{Cascade of Blades}

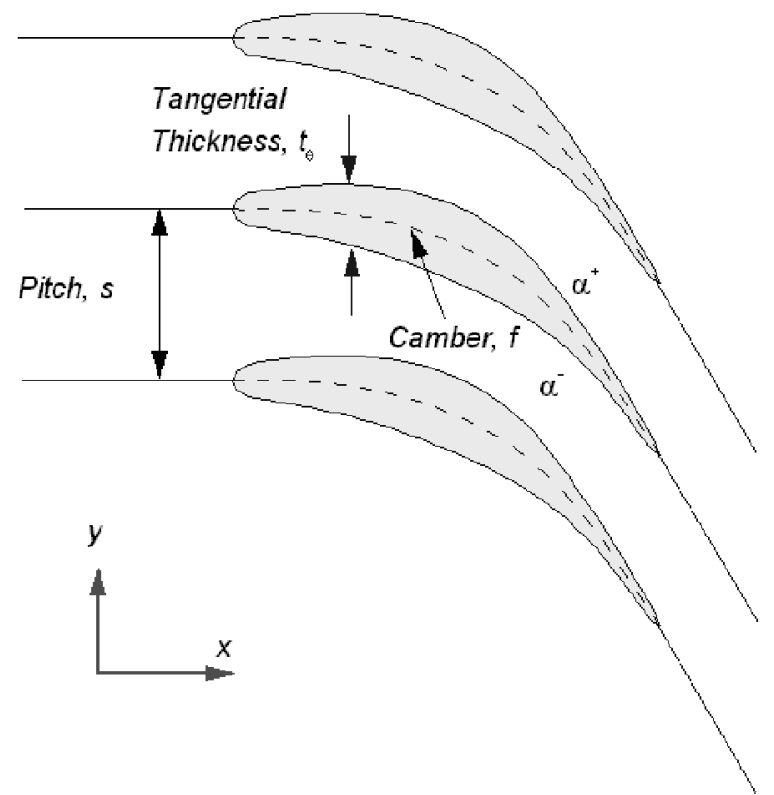

Fig. 4 Definition of blade cascade geometry 
The blade-averaged value is indicated with the subscript bl and the blade difference value with the notation $\Delta$ :

$$
\begin{aligned}
& V_{x_{\mathrm{bl}}}=\frac{1}{2}\left(V_{x}^{+}+V_{x}^{-}\right), \quad V_{y_{\mathrm{bl}}}=\frac{1}{2}\left(V_{y}^{+}+V_{y}^{-}\right), \\
& \Delta V_{x}=V_{x}^{+}-V_{x}^{-}
\end{aligned}
$$

Equation (10) can be expressed accordingly as

$$
V_{x_{\mathrm{bl}}}^{0} \frac{\mathrm{d} f^{0}}{\mathrm{~d} x}+\frac{1}{4} \frac{\mathrm{d} t_{\theta}}{\mathrm{d} x} \Delta V_{x}^{0}=V_{y_{\mathrm{bl}}}^{0}
$$

To approximate for the new camber geometry, $f^{1}$, the correction term given by $\tilde{V}_{y}^{*}-\tilde{V}_{y}^{0}$ is introduced in the right-hand side of the equation to give

$$
V_{x_{\mathrm{bl}}}^{0} \frac{\mathrm{d} f^{0}}{\mathrm{~d} x}+\frac{1}{4} \frac{\mathrm{d} t_{\theta}}{\mathrm{d} x} \Delta V_{x}^{0}=V_{y_{\mathrm{bl}}}^{0}+\left(\tilde{V}_{y}^{*}-\tilde{V}_{y}^{0}\right)
$$

Then by subtracting equation (12) from (13), the necessary change in the cascade shape (i.e. $f^{1}-f^{0}$ ) is given by

$$
V_{x_{\mathrm{b} 1}}^{0} \frac{\mathrm{d}\left(f^{1}-f^{0}\right)}{\mathrm{d} x}=\tilde{V}_{y}^{*}-\tilde{V}_{y}^{0}
$$

Equation (14) is then integrated numerically to give the new camber geometry, $f^{1}$. Once $f^{1}$ is obtained, the new profile is defined by adding the specified tangential thickness symmetrically to the new camber line. The design solution is considered to have converged at the $n$th iteration when $\tilde{V}_{y}^{*}-\tilde{V}_{y}^{n}$ reaches a certain tolerance. This approach is therefore similar to that used by Soulis [29], which is based on potential flow for the design of blades with zero thickness.

\subsection{Prediction of initial geometry}

In practical design attempts, the design task usually concerns improving a known blade. In such cases, the design procedure can start with the known geometry as the initial shape. In a new design attempt when the initial blade shape is not known, an estimation of the initial blade shape needs to be made. In such cases, the estimation is made on the basis of the specified target $\tilde{V}_{y}^{*}$ distribution using a simplified version of equation (9), which is

$$
\frac{\mathrm{d} f^{0}}{\mathrm{~d} x}=\frac{\tilde{V}_{y}^{*}}{V_{x}^{\text {est }}}
$$

where $V_{x}^{\text {est }}$ is determined from the required mass flowrate. Once the initial camber geometry is defined, the cascade geometry is readily defined by prescribing the fixed tangential thickness profile.

It is noted that the current method of approximating the initial cascade shape is a simple one. Nevertheless, it only needs to be a rough approximation and, in all the test cases encountered, equation (15) has proven to be adequate in providing a quick and reasonable initialization for the design procedure.

\subsection{Convergence criteria}

In the inverse methodology, the sequence of performing flow analysis and making the corresponding blade update forms the main design cycle.

Geometrical computations of the new cascade shape are performed after the convergence of each flow analysis. The convergence criteria used for the flow calculation are that of percentage mass error and root mean square error on velocity of the flow field. The number of iterations required in obtaining each direct solution is typically about 2000 and decreases as the design converges towards the final geometry. In the context of the design procedure, convergence is obtained when the computed mass-averaged tangential velocity distribution is sufficiently close to the target distribution $(<1.0$ per cent) and when the modification of the blade is sufficiently small (of order $10^{-3}$ ) as compared with the axial chord length.

\section{THE DISTRIBUTION PARAMETRIZATION}

The value of $\tilde{V}_{y}$ at the leading and trailing edges of a cascade is determined from Euler's equation for turbomachinery. Furthermore, since the jump in pressure across the blade is given by equation (4), the derivative of $\tilde{V}_{y}$ in the axial direction (i.e. $\mathrm{d} \tilde{V}_{y} / \mathrm{d} x$ ) must be set to zero at the trailing edge in order to satisfy the Kutta condition.

Taking into account these constraints, $\tilde{V}_{y}$ is represented by a cubic $B$-spline curve. Using a control polygon with coordinates $\left\{b_{i}, i=0, \ldots, n\right\}$, the cubic $B$-spline curve is defined by

$$
\tilde{V}_{y}=\sum_{i=1}^{n} b_{i} B_{i}(t)
$$

where $\left\{B_{i}(t)\right\}$ are the cubic $B$-spline basis functions with the set of knots $\left(t_{0}, \ldots, t_{n}\right)$ and $b_{i}=b_{i_{x}}-b_{i_{v}}$. Because the basis functions have compact local support, changing a point in the corresponding control polygon only changes the course of the curve locally and the influence of each control point can be pinpointed precisely. It is also easy to introduce additional control points; therefore fewer control points may be used initially to search for an improved design. In practice, if the set of knots is chosen to be the integer set $(0, \ldots, n)$, equation (16) becomes the uniform $B$-spline curve [30].

In order to satisfy the Kutta condition at the trailing edge and to control the loading distribution at the leading edge, the second and the penultimate control points are fixed to give the required slope. Also, since $\tilde{V}_{y}$ is given at the leading and trailing edges by Euler's equation for turbomachinery, the first control point is therefore fixed there. Moreover, two extra artificial control points are used, one at each end, 
extended by an equal distance and an equal slope to the corresponding segment. A typical $\tilde{V}_{y}$ together with its control polygon is shown in Fig. 5. The loading distribution can be sought by differentiating $\tilde{V}_{y}$.

In practice, although the cubic $B$-spline ensures the continuity and differentiability of the loading distribution, it does not control the number of points of inflection in $\tilde{V}_{y}$. In some cases, the loading $\left(\mathrm{d} \tilde{V}_{y} / \mathrm{d} x\right)$ distribution might have excessive numbers of turning points which can in turn give rise to an impractical blade profile. In order to avoid this, the total curvature of the mass-averaged tangential velocity distribution is controlled via

$$
\int_{x_{\mathrm{LE}}}^{x_{\mathrm{TE}}}\left|\tilde{V}_{y}^{\prime \prime}\right| \mathrm{d} l<\kappa_{\varepsilon}
$$

where $l$ is the arc length and $\kappa_{\varepsilon}$ is the predefined constraint constant.

\section{THE SIMULATED ANNEALING ALGORITHM}

In searching for the optimal distribution, $\left\{b_{i}\right\}$, in the parametrization equation (16) is selected randomly between two given bounds $\left[b_{1}, b_{\mathrm{u}}\right]$ and given as an input into the inverse design method to generate the cascade shape.

In order to manipulate the sequence of randomly generated $\left\{b_{i}\right\}$ correctly, the SA algorithm is needed. In the algorithm, the variables $T, \varepsilon, N_{\mathrm{c}}$ represent respectively the current temperature, cooling speed and number of cooling steps. For each cooling step, a total of $N$ perturbations are executed while, for each perturbation, either one or all of the $n$ control points are randomly selected for perturbation. If the perturbed distribution calculated from the set of perturbed control points $b^{\prime}$ violates the curvature constraint, the control points are regenerated. Following successful regeneration of the specification from the control points, the inverse design cycle can commence.

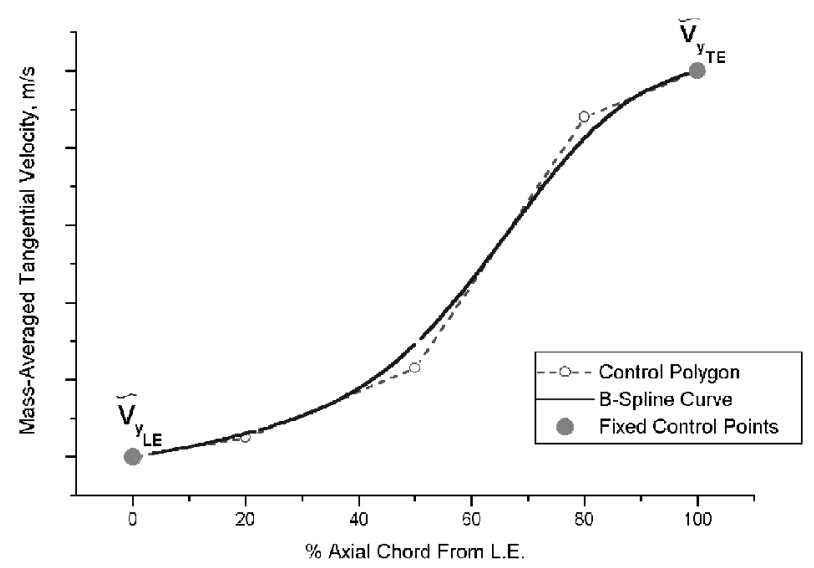

Fig. 5 A typical control polygon and the corresponding cubic $B$-spline curve
In the proposed exploratory optimization procedure, a good number of inverse design runs are performed systematically to determine the corresponding cascade shapes of the generated $\tilde{V}_{y}$ specifications. In the current implementation, a database of the generated designs (and their flow solutions) is set up and is updated automatically throughout the entire procedure. In each inverse calculation, the candidate (and its flow solution) in the database having the closest match in terms of its $\tilde{V}_{y}$ control polygon points to the current specification is used to start the current inverse design calculation. This approach significantly reduces the computational time required.

On convergence of each inverse design run, the new blade and its flow field are checked. If the loss of the new geometry is less than the previous one, the new $\tilde{V}_{y}$ distribution is accepted; otherwise, the acceptance of the perturbed distribution is dependent on a Boltzmann probabilistic distribution chosen as

$$
\operatorname{prob}=T \exp \left[\frac{\operatorname{loss}(b)-\operatorname{loss}\left(b^{\prime}\right)}{T}\right]
$$

If the generated random number is less than prob, the new solution is accepted. The temperature $T$ of the system is modified by multiplying it by $\varepsilon$ after $N$ searches. This cooling process is repeated until the temperature of the system reaches the final value.

The algorithm begins with a relatively high temperature $T$ in order to avoid premature entrapment at local minima. As $T$ starts to decrease, the chance of rejecting an upward move in the cost function will increase. When the temperature becomes very low, the effort to reduce the cost function further will be met with limited success, which signals that the annealing procedure should be terminated. As the number of control points increases, a large number of searching steps $N$ may be needed in order to converge to the global solution. It should be pointed out that there is no rigorous rule governing the selection of the initial temperature, the cooling speed $\varepsilon$ and the number of searching steps $N$. These annealing parameters themselves have to be optimized by experience with regard to convergence and computer speed. For the present application, the number of cooling step $N_{\mathrm{c}}$ is chosen to be $10, N$ is 300 , initial $T$ is 0.5 and $\varepsilon$ is 0.75 .

In the following, the algorithm is summarized. Note that all uniformly distributed random numbers are generated using an established routine [31]. The expression $\{a, b\}$ is used to denote an integer number ranging from $a$ to $b$, while the square brackets indicate a real continuous value.

\section{Initialization}

Get an initial $\tilde{V}_{y}$ distribution and set $b_{1}, b_{\mathrm{u}}, \kappa_{\varepsilon}$ for $T=0.5, \varepsilon=0.75, N_{\mathrm{c}}=10$ and $N=300$.

$$
\begin{aligned}
& \text { Cooling } \\
& \quad \text { For cycle }=1, \ldots, N_{\mathrm{c}}
\end{aligned}
$$




\section{Searching}

For step $=1, \ldots, N$

1. Index $=$ random $\{0,1\}$.

2. If index $=1, b_{i_{y}}^{\text {new }}=\operatorname{random}\left[b_{1}, b_{\mathrm{u}}\right]$, $i=1, \ldots, n$.

3. If index $=0, p=\operatorname{random}\{1, \ldots, n\}$ and $b_{p_{y}}^{\text {new }}=$ random $\left[b_{1}, b_{\mathrm{u}}\right]$.

4. If curvature $>\kappa_{\varepsilon}$, goto 1 .

5. Search through the database for $\min _{k}\left\|b_{k}-b^{\text {new }}\right\|_{2}$ and input as initial guess.

6. Calculate $\ddot{a}=\operatorname{loss}\left(b^{\text {new }}\right)-\operatorname{loss}\left(b^{\text {old }}\right)$. If $\delta<0$, then $b^{\text {old }}=b^{\text {new }}$, else if random $[0,1]<T$ $\exp (-\delta / T), b^{\text {old }}=b^{\text {new }}$.

7. Include $b^{\text {new }}$ in the database if $\min _{k}\left\|b_{k}-b^{\text {new }}\right\|_{2}$ is larger than a threshold value.

Update $T=\varepsilon T$.

\section{NUMERICAL RESULTS}

\subsection{Validations: reproduction of UTRC rotor cascade}

The inverse method is first tested with the UTRC experimental mid-span rotor profile as discussed earlier in Section 2.2. The validation aims to reproduce the original profile starting with a different geometry in order to verify the accuracy of the method. The mass-averaged tangential velocity distribution of the original cascade is first obtained from the flow analysis performed earlier and imposed as the design specifications for the inverse design procedure.

Figure 6 shows the starting initial blade and the progressive modifications carried out before arriving at the final geometry. Figure 7 illustrates the comparison between the imposed and final converged distribution of $\tilde{V}_{y}$.

The reproduced profile closely matches the original shape with maximum geometrical difference of less than 1 per cent, and thus the accuracy of the method is adequately verified.

\subsection{Compressor rotor inverse design and optimization}

The working of the proposed inverse design procedure is demonstrated first in the design of a generic transonic compressor rotor cascade. The rotor profile is subjected to a constant rotating tangential velocity set at $240 \mathrm{~m} / \mathrm{s}$. Other flow parameters are as follows: inlet flow angle $\beta_{1}=0.0^{\circ}$, inlet stagnation conditions $T_{0_{1}}=300 \mathrm{~K}$ and $P_{0_{1}}=111896 \mathrm{~Pa}$ and exit static pressure $P_{2}=111958 \mathrm{~Pa}$.

As a preliminary study prior to the application of the optimization routine, it is first attempted to vary the imposed $\tilde{V}_{y}$ distribution on the basis of experience and to apply the inverse design method to solve for the corresponding profile. The inverse design procedure starts with an initial geometry whose camber is predicted via equation (15). Its tangential thickness follows a double circular arc distribution, which has a maximum of 9 per cent of the axial chord at mid-chord and the cascade has a pitch-to-chord ratio of 0.83 . The target specific head rise $(\Psi)$ is specified to be 0.4

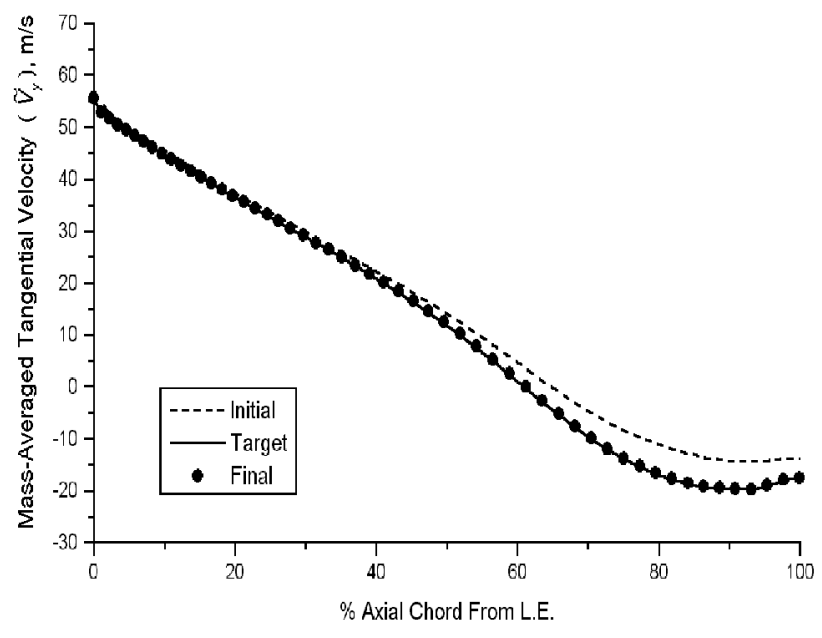

Fig. 7 Validation of inverse method: target and final massaveraged tangential velocity distributions

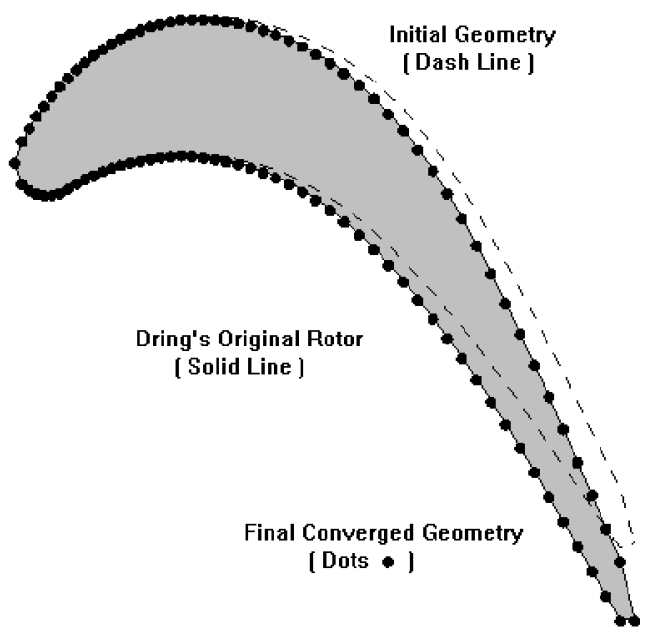

Fig. 6 Validation of inverse method: reproducing UTRC rotor profile 
corresponding to zero inlet swirl and exit tangential velocity of $96 \mathrm{~m} / \mathrm{s}$.

Two main features of the initial cascade stand out (see Fig. 8). The first is the presence of a very strong shock standing normal across the passage and impinging on both surfaces of the cascade. The second is the strong diffusion along the suction surface, aft of the blade following the shock. Both features are clearly registered in the loading distribution (see middle right-hand plot in Fig. 8): the shock formation as a sharp gradient between 25 and 65 per cent axial chord position and the diffusion aft of the blade as a steep unloading from the peak position. The loading distribution is therefore as good a gauge of such flow features as traditional properties such as surface pressure or velocity
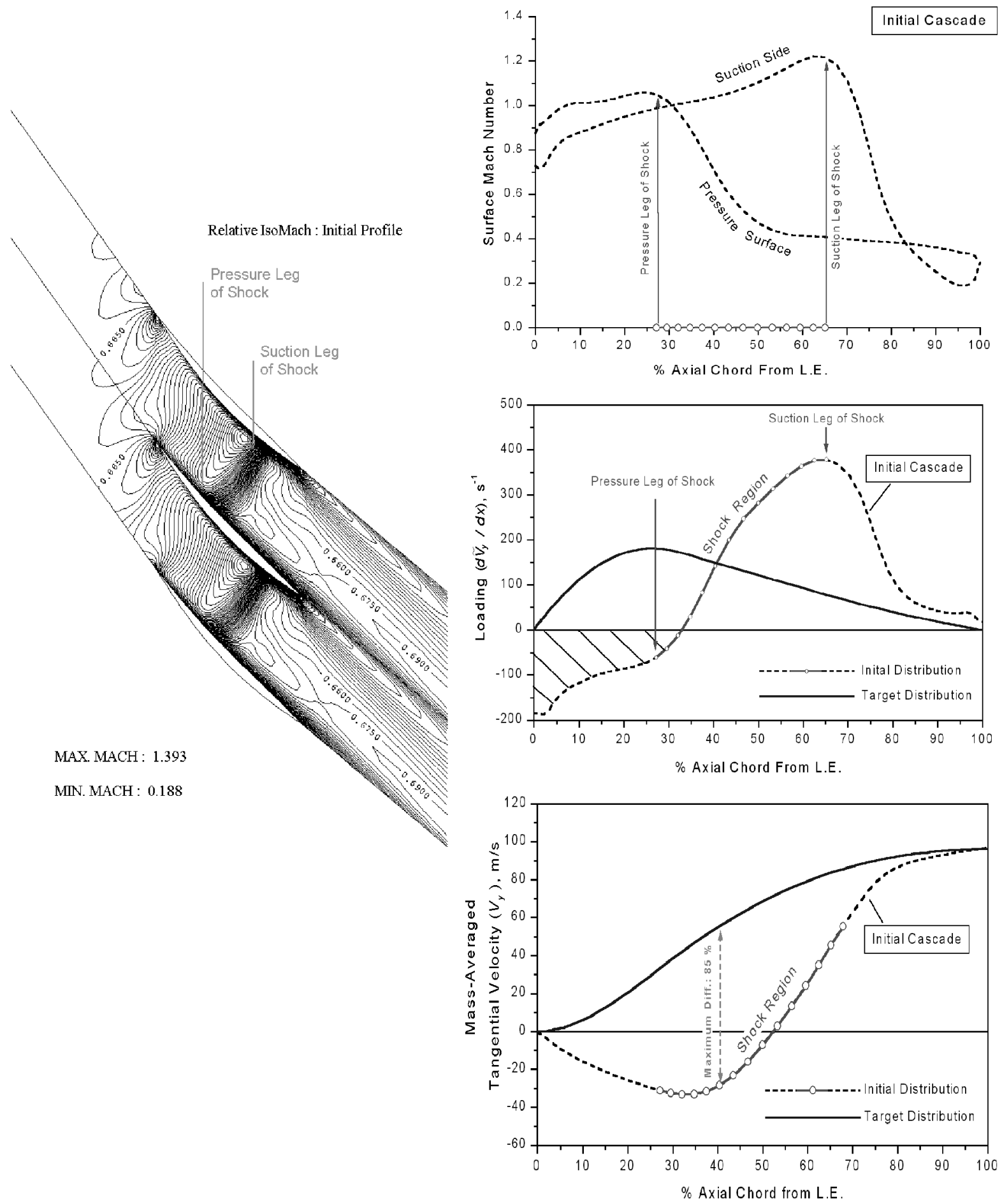

Fig. 8 Progressive changes in mass-averaged tangential velocity distribution 
and is thus suitable as a design parameter in transonic design.

As the design computation progresses, its effects on the flow are traced. These are directly brought about by the changing geometry whose loading distribution is converging towards the specified shape as shown in Fig. 9, where the sequence of changes may be visualized.

As depicted in Figs 9 and 10, when the loading distribution is modified from being mainly rear loaded to fore loaded, the position of the shock is also moved from near the trailing edge towards the front, near the leading edge. In addition, it is also noted that the decreasing peak load and gradient lead to a weakening shock whose effects are clearly illustrated by the diminishing shock patterns, also in Fig. 10 .

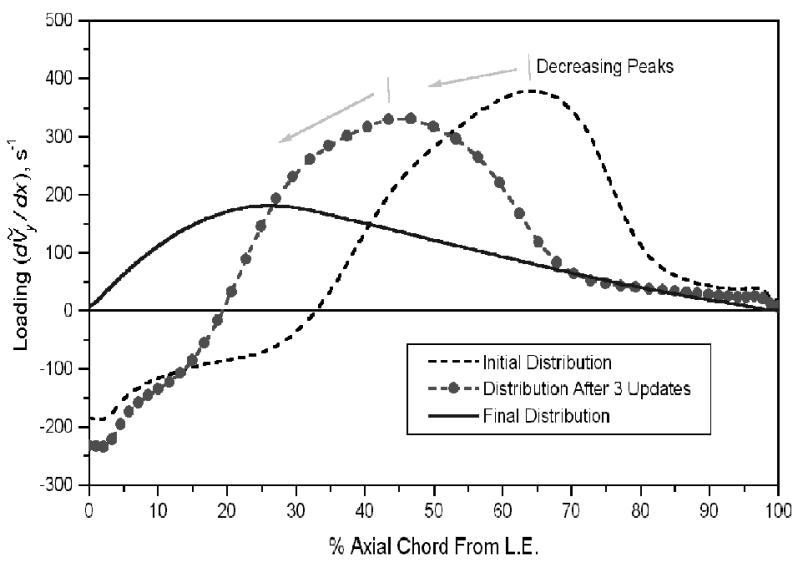

Fig. 9 Progressive changes in loading distribution
This design attempt demonstrates the successful working of the inverse method to achieve the specified $\tilde{V}_{y}$ distribution. In coupling the inverse methodology with the SA algorithm, the aim is to search for a better $\tilde{V}_{y}$ distribution. It is noted that, in this example and the next, the tangential thickness distributions of the cascade are held fixed in the entire optimization procedure.

From the experience gathered in using the inverse method for the initial attempt discussed above, it is found necessary to avoid a large negatively loaded region at the leading edge as this corresponds to a low work level and the formation of shock. This is introduced as a constraint in the optimization procedure. It is emphasized that the constraint is not a restriction of the method but simply to demonstrate the possibility of incorporating any prior knowledge or experience to apply the optimization procedure within a relevant domain of interest. In the authors' view, this capability is invaluable since it allows established design knowledge to be incorporated into the proposed procedure to reduce unnecessary costly 'blind' searching.

Figure 11 shows the comparison between the inverse designed fore-loaded design, the final optimized profile and an intermediate optimized profile. Comparisons of the surface Mach number distributions are shown in Fig. 12. Hobbs and Weingold [32] discuss the usefulness in adopting a 'ski-jump' surface Mach number distribution for shockfree or supercritical designs. The primary mechanism in the 'ski-jump' distribution is to maintain high but smooth flow acceleration near the leading edge where the boundary layer is still thin and to allow gradual deceleration of the flow aft of the blade profile. It is interesting to note that the
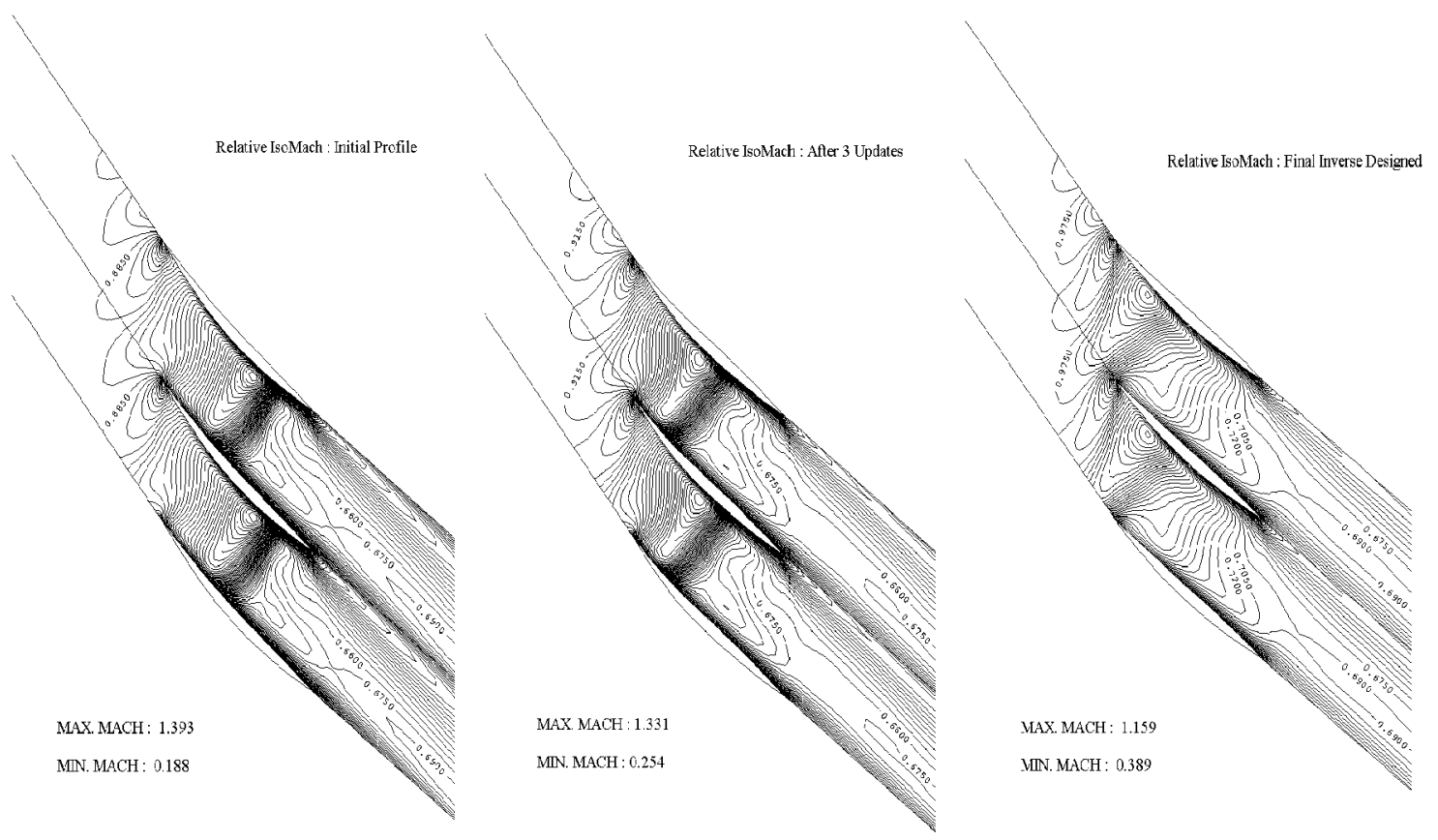

Fig. 10 Relative Mach number contours: initial, intermediate and final (contour interval: 0.015) 


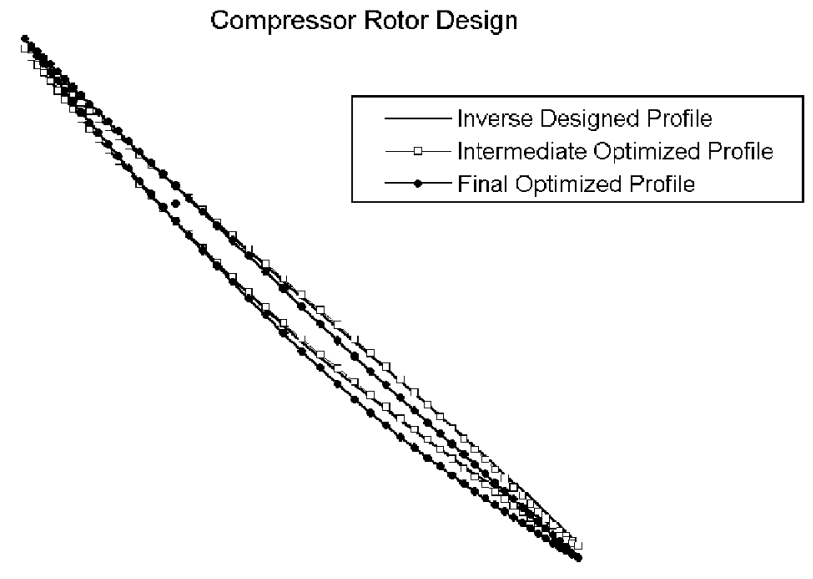

Fig. 11 Inverse designed, intermediate optimized and final optimized blade geometries

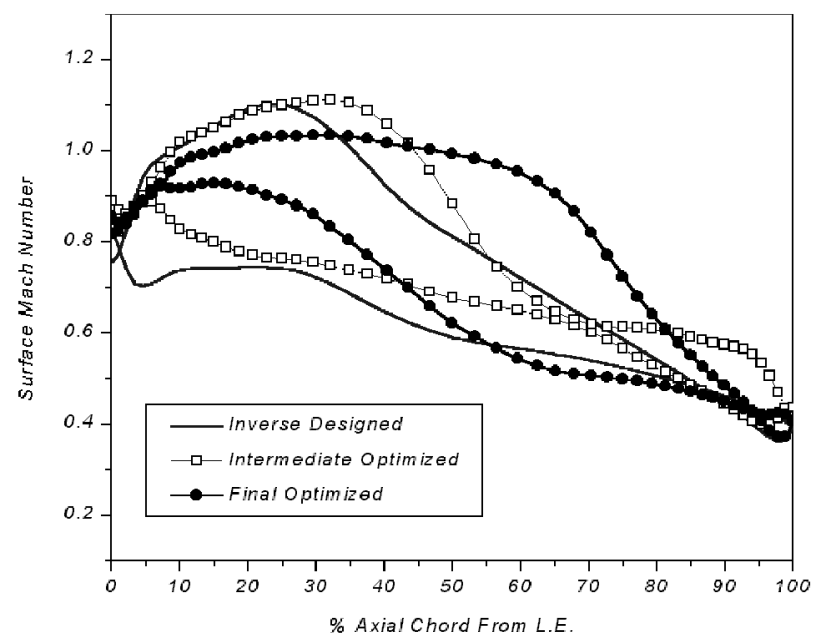

Fig. 12 Inverse designed, intermediate and final optimized surface Mach number distributions

optimized profiles (intermediate and final) both exhibit this form in their surface Mach number distributions, affirming the study in reference [32]. More interestingly, the additional hump in the suction Mach number distribution of the intermediately optimized cascade, which effectively gives a 'double ski-jump' shape, has enhanced the performance of the original profile. The surface velocity on the suction surface of the intermediate cascade is allowed to rise to a higher peak than the datum profile, but the diffusion rate on the suction surface is moderated with a slight acceleration at the 65 per cent axial chord position. The final optimized cascade exhibits a gentle velocity acceleration at the front of the profile; the peak velocity is maintained over a large proportion on the suction surface (about 45 per cent of the axial chord length) before diffusion takes place. The final profile therefore avoids unnecessary acceleration at the front as well as sharp diffusion of the flow through its cascade,

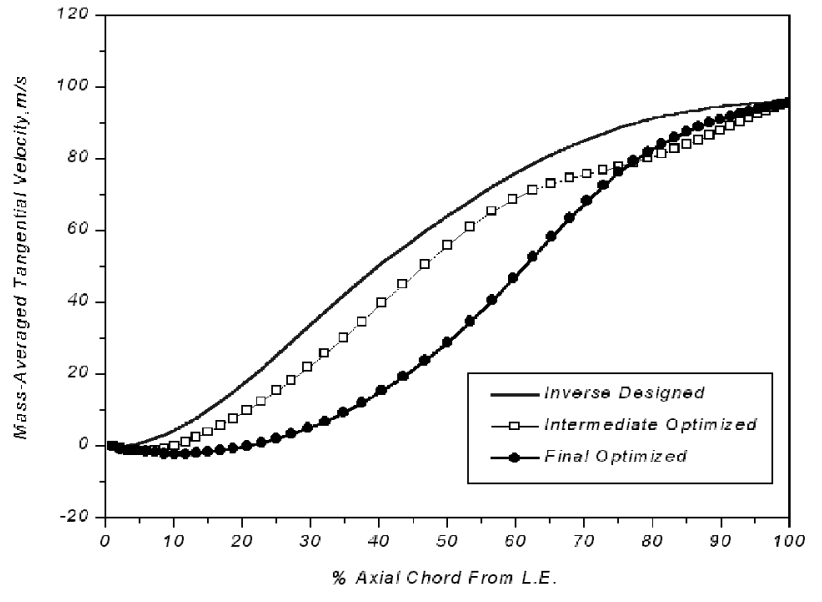

Fig. 13 Inverse designed, intermediate and final optimized mass-averaged tangential velocity distributions

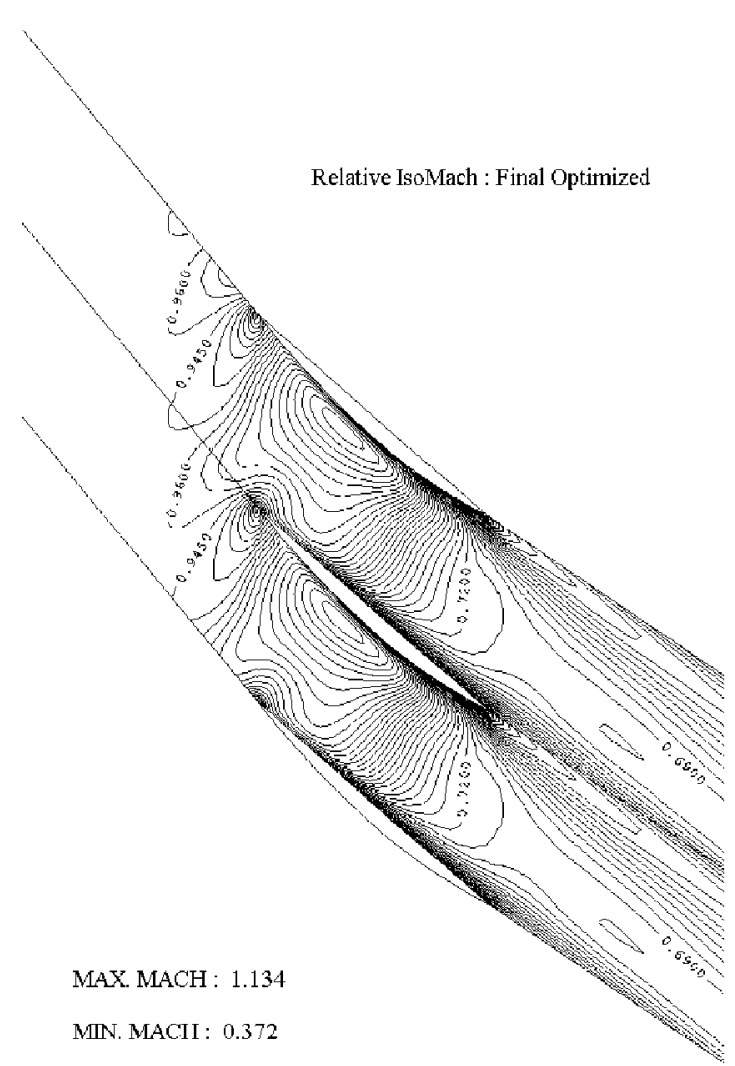

Fig. 14 Final optimized geometry: iso-Mach contours and velocity vectors

which results in the lowest overall loss. The corresponding mass-averaged tangential velocity distributions for all three designs may be examined in Fig. 13.

The Mach number contours of the final optimized blade profile are given in Fig. 14. The flow field can be observed 
to be smoothly varying where the supersonic patch near the leading edge is developed in the absence of a strong shock formation and no abrupt flow deceleration further downstream.

Entropy loss calculation, $\exp (-\Delta S / R)$, is used as the measure of loss in this work; here, a value of 1.0 represents no thermodynamic irreversibility and a decreasing value indicates higher losses. There is a considerable improvement in the inverse designed blade shape where the initial loss value of 0.941 is improved to 0.978 . Execution of the optimization produced a further improvement of 18 per cent for the intermediate optimized profile after 158 perturbations and 23 per cent for the final optimized blade shape at the 347th iteration. Clearly, the iteration can be stopped immediately after the shock has been eliminated. Here, the computation is performed to the end simply to demonstrate that this is the best possible solution resulting from the run.

\subsection{Turbine stator optimization}

The proposed optimization procedure is demonstrated next in the design of a stator blade in a transonic flow regime. The blade is prescribed with the British $\mathrm{C} 4$ series thickness with a maximum thickness of 12.5 per cent of the axial chord. The cascade is arranged with a solidity of 1.0 and zero inlet flow angle. Flow pressure ratio $P_{2} / P_{0_{1}}$ is fixed at 0.55 and the design requirement is set to produce zero mass-averaged tangential velocity at the inlet and $200 \mathrm{~m} / \mathrm{s}$ at the outlet.

The initial blade used for the optimization procedure is first derived by the inverse method whose mass-averaged tangential velocity specification is generated via a threesegmented algorithm proposed by Zangeneh et al. [33]. The algorithm generates the initial specification on the basis of the required inlet and outlet values of $\tilde{V}_{y}$ and a user-specified slope that controls the rate of change along the middle segment. The generated $\tilde{V}_{y}$ and loading distribution are shown as the full curves in Figs 15 and 16 respectively.

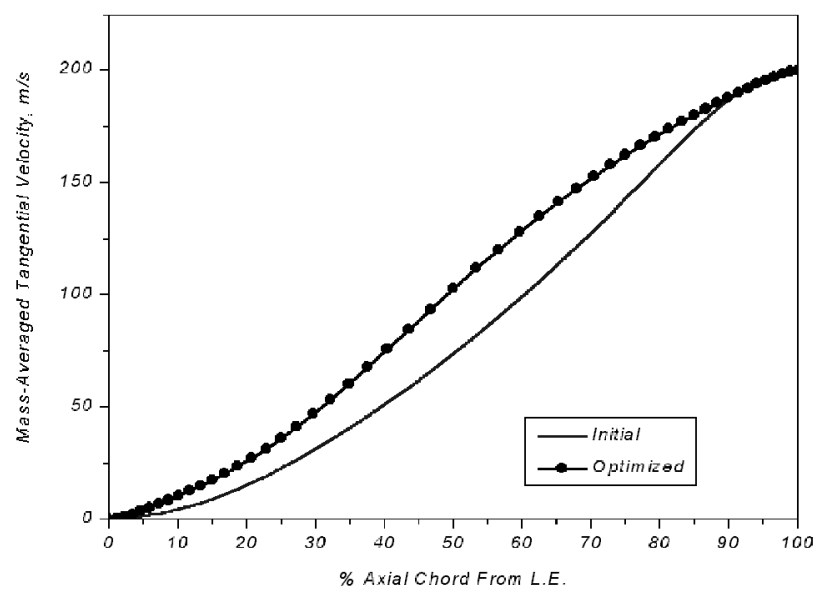

Fig. 15 Initial and optimized mass-averaged tangential velocities
The optimization procedure is used next to improve the performance of this initial design. In this case, the datum design was significantly modified to give finally the optimal aerodynamic performance (see Fig. 17). The optimized loading distribution has a middle-loaded characteristic (represented by the full curve and circles in Fig. 16) with a gentle rate of change as compared with that of the initial cascade. The resulting effect is a weakened shock wave formation and Fig. 18 shows the plots of the surface Mach number distribution of the two cascades. The suction surface Mach distribution (see full curve) of the initial cascade shows abrupt acceleration and deceleration of flow near its leading and trailing edges. In contrast, controlled surface flow variations are observed for the optimized profile as represented by the curve with solid circles.

Entropy generation, $\exp (-\Delta S / R)$, shows a significant improvement in loss from an initial value of 0.952 to 0.9775 after 1472 perturbations in the optimization process.

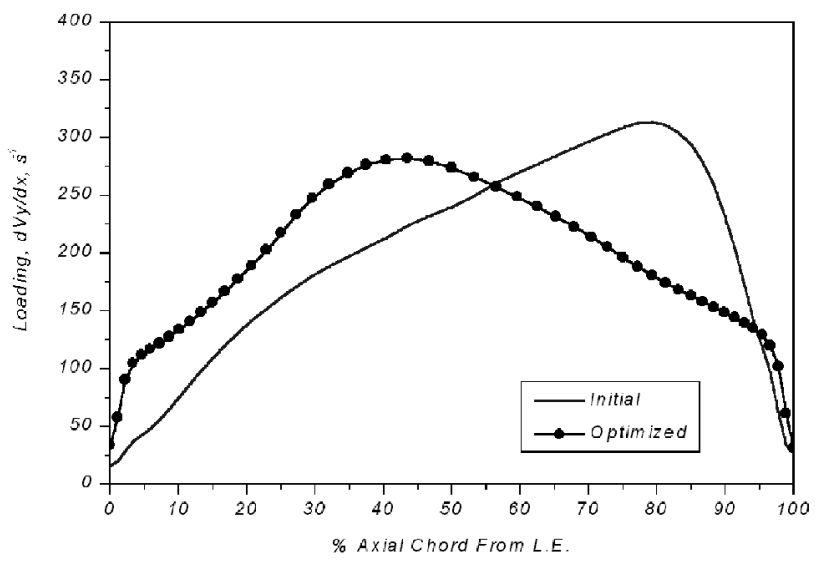

Fig. 16 Initial and optimized loading distributions

Turbine Stator Design

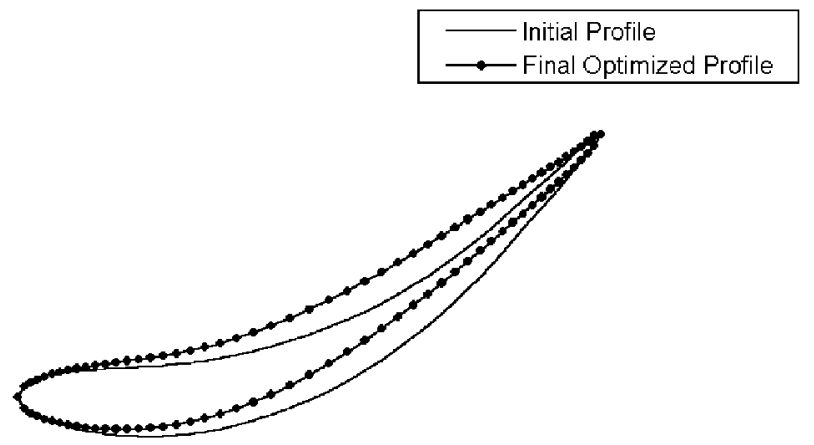

Fig. 17 Plot of initial and final blade geometries 


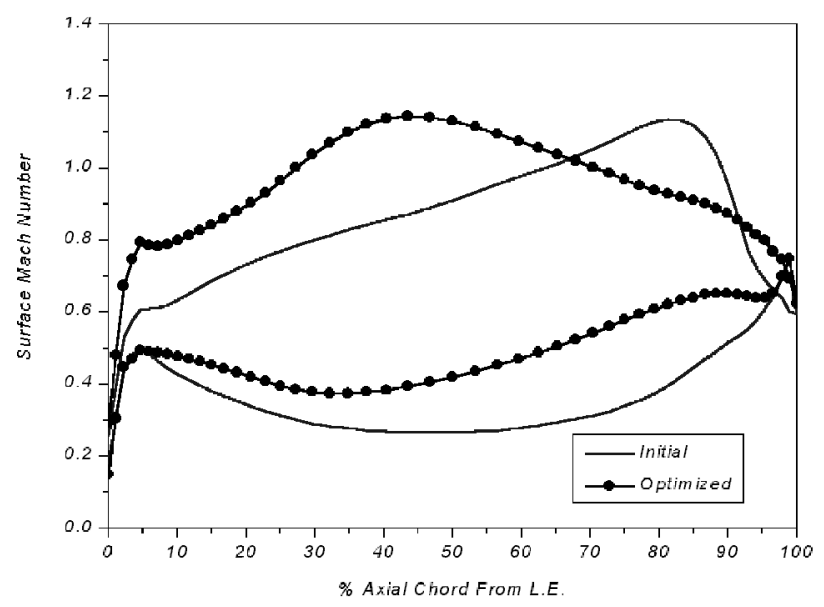

Fig. 18 Surface Mach number distributions

\section{CONCLUSION}

The feasibility of an automated inverse optimization design procedure with the capability to access known designs, stored in the form of a database, is exhibited. Using two generic transonic cascades, it is shown that optimal designs of the cascades can be readily achieved by exploiting in combination the systematic approach of a new inverse methodology and the stochastic nature of the SA algorithm.

In the inverse method module, the target swirl velocity distribution is parametrized using a cubic $B$-spline curve and is regenerated by the randomly biased searches using SA. The entire computation requires minimal human intervention except during initial set-up where constraints based on existing knowledge may be imposed to restrict the search for the optimal performance to a specified domain of interest. To improve computational performance further, the procedure is set up with the use of a solution database (e.g. previously generated) to speed up the calculation.

Two generic transonic designs have been presented, one for turbine stator and one for compressor rotor. The proposed algorithm has been applied very successfully to both cases with the optimum cascades showing obvious aerodynamic improvement over the datum designs. Loss reductions in the region of 20 per cent have been achieved in both test cases.

The results show conclusively the potential of the proposed optimization procedure for practical design usage. The automated procedure may be applied to generate a completely new optimum cascade design or to improve on existing designs. In the latter case, the ability of the procedure to include useful prior knowledge as constraints to confine design searches within the user-specified domain of relevance is most useful. It is envisaged that the proposed inverse optimization method in three dimensions may be a very useful tool for blade designers. The extension of the proposed procedure to three dimensions is now in progress. It is also noted that the current optimization routine may be employed with other inverse methodologies based on different design parameters. Collective studies on the results produced by different inverse methods may give rise to valuable insights to the characteristics of optimum designs, in terms of different design parameters.

\section{ACKNOWLEDGEMENT}

This research and development programme was funded by Ebara Research Company Limited (Japan). The authors gratefully acknowledge the financial and technical support given by the company during the course of the project.

\section{REFERENCES}

1 Meauze, G. An inverse time marching method for the definition of cascade geometry. J. Engng Pwr, 1982, 104, 650-656.

2 Hart, M. and Whitehead, D. S. A design method for 2D cascades of turbomachinery blades. Int. J. Numer. Meth. Fluids, 1987, 7, 1363-1381.

3 Leonard, O. and Van Den Braembussche, R. A. Design method for subsonic and transonic cascade with prescribed Mach number distribution. ASME paper 91-GT-18, 1991.

4 Zangeneh, M. A compressible three-dimensional design method for radial and mixed flow turbomachinery blades. Int J. Numer. Meth. Fluids, 1991, 13, 599-624.

5 Demeulenaere, A. and Van Den Braembussche, R. A. Threedimensional inverse method for turbomachinery blading design. ASME paper 96-GT-39, 1996.

6 Dulikravich, G. S. and Baker, D. P. Aerodynamic shape inverse design using a Fourier series method. AIAA paper 99-0185, 1999.

7 Denton, J. D. The use of a distributed body force to simulate viscous effects in 3D flow calculations. ASME paper 86-GT$144,1986$.

8 Giles, M. B. and Drela, M. Two dimensional transonic aerodynamics design method. Am. Inst. Aeronaut. Astronaut. J., 1987, 9, 1199-1206.

9 Dang, T. and Isgro, V. Euler-based inverse method for turbomachine blades, Part I: 2-D cascades. Am. Inst. Aeronaut. Astronaut. J., 1995, 33(12), 2309-2315.

10 Dang, T. Inverse method for turbomachine blades using shockcapturing techniques. AIAA paper 95-2465, 1995.

11 Tiow, W. T. and Zangeneh, M. A viscous transonic inverse design method for turbomachinery blades, Part I: 2D cascades. ASME paper 98-GT-125, 1998.

12 Zangeneh, M., Goto, A. and Harada, H. On the design criteria for suppression of secondary flows in centrifugal and mixed flow impellers. ASME paper 97-GT-393, 1997.

13 Ashihara, K. and Goto, A. Improvements of pump suction performance using 3D inverse design method. ASME paper FEDSM99-6846, 1999.

14 Black, S. D. Integrated lifting-surface/Navier-Stokes design and analysis methods for marine propulsors. $\mathrm{PhD}$ thesis, Massachusetts Institute of Technology, 1997.

15 Yiu, K. F. C. and Zangeneh, M. On the simultaneous design of blade and duct geometry of marine ducted propulsors. J. Ship Res., 1998, 42(4), 274-296. 
16 Tiow, W. T. and Zangeneh, M. A three-dimensional viscous transonic inverse design method. ASME paper 2000-GT-525, 2000.

17 Van Egmond, J. A. Numerical optimization of target pressure distributions for subsonic and transonic airfoil design. In Computational Methods for Aerodynamic Design (Inverse) and Optimization, AGARD-CP-463, Loen, Norway, 1989.

18 Van den Dam, R. F., van Egmond, J. A. and Slooff, J. W. Optimization of target pressure distributions. In Inverse Methods in Airfoil Design for Aeronautical and Turbomachinery Applications, AGARD-R-780, 1990.

19 Yiu, K. F. C. and Zangeneh, M. A 3D automatic optimization strategy for design of centrifugal compressor impeller blades. ASME paper 98-GT-128, 1998.

20 Kirkpatrick, S., Gelatt, C. D. and Vecchi, M. P. Optimization by simulated annealing. Science, 1983, 220, 671-680.

21 Obayashi, S. and Takanashi, S. Genetic optimization of target pressure distributions for inverse design methods. Am. Inst. Aeronaut. Astronaut. J., 1996, 34(5), 881-886.

22 Metropolis, N., Rosenbluth, A., Rosenbluth, M., Teller, A. and Teller, E. Equation of state calculations by fast computing machines. J. Chem. Physics, 1953, 21, 1087-1092.

23 Cerny, V. Thermodynamical approach to the traveling salesman problem: an efficient simulation algorithm. J. Optimization Theory Applic., 1985, 45, 41-45.

24 Denton, J. D. and Singh, U. K. Application of numerical methods to flow calculations in turbomachines. In VKI Lecture Series 1979-7, 1979.
25 Hall, M. G. Cell-vertex multigrid schemes for solution of the Euler equations. In Conference on Numerical Methods for Fluid Dynamics (Eds K. W. Morton and M. J. Baines), Reading, 1986.

26 Jameson, A., Schmidt, W. and Turkel, E. Numerical solutions of the Euler equations by finite volume method using Runge Kutta time stepping schemes. AIAA paper 81-1259, 1981.

27 Dring, R. P., Joslyn, H. D., Hardin, L. W. and Wagner, J. H. Turbine rotor-stator interaction. ASME paper 82-GT-3, 1982.

28 Tan, C. S., Hawthorne, W. R., McCune, J. E. and Wang, C. Theory of blade design for large deflections, Part II: annular cascade. J. Engng Gas Turbines Pwr, 1984, 106, 346-353.

29 Soulis, J. V. Thin turbomachinery blade design using a finitevolume method. Int. J. Numer. Meth. Engng, 1985, 21, 19-36.

30 Coons, S. A. Surface patches and B-spline curves. In Computer Aided Geometric Design (Eds R. Barnhill and R. Riesenfeld), 1974 (Academic Press, New York) pp. 1-16.

31 Press, W. H., Teukolsky, S. A., Vetterling, W. T. and Flannery, B. P. Numerical Recipes in Fortran: The Art of Scientific Computing, 2nd edition, 1992 (Cambridge University Press, Cambridge).

32 Hobbs, D. E. and Weingold, H. D. Development of controlled diffusion airfoils for multistage compressor application. $J$. Engng Gas Turbines Pwr, 1984, 106, 271-278.

33 Zangeneh, M., Goto, A. and Takemura, T. Suppression of secondary flows in a mixed-flow pump impeller by application of three-dimensionalinverse design method: Part I-design and numerical validation. J. Turbomachinery, 1996, 118, 536-551. 